\title{
An Efficient Non-blind Watermarking Scheme for Color Images using Discrete Wavelet Transformation
}

\author{
Nagaraj V. Dharwadkar \\ Computer Science \& Engineering \\ National Institute of Technology \\ Warangal, India.
}

\author{
B. B. Amberker \\ Computer Science \& Engineering, \\ National Institute of Technology \\ Warangal, India.
}

\begin{abstract}
In this paper we present a new invisible robust non blind watermarking scheme. The proposed scheme embeds the monochrome $(\log 0)$ watermark into the high and middle $\{\mathrm{HL}, \mathrm{LH}, \mathrm{HH}\}$ frequency bands of luminance channel of the color image. The Red (R), Green (G) and Blue (B) channels of color image are transformed into Luminance (Y), Intensity (I) and Hue (Q) Channels, the Luminance channel of color image is decomposed using Discrete Wavelet Transformation (DWT), then the high and middle frequency components of this Luminance are used to embed the watermark. To increase the detection speed and efficiency of algorithm, the location of modified high and middle frequency components are stored into key array. Use of this key array improves the speed of the extraction algorithm. The experimental results show that the watermark is robust against different types of attacks like image cropping, image filtering, image Compression and Image transformations. Further, the results show that the performance of our scheme is superior to other similar techniques.
\end{abstract}

\section{Categories and Subject Descriptors}

D.3.3 [Programming Tools]: MATLAB Constructs and Features - abstract data types, control structure and Image Processing tool.

\section{General Terms}

Graphics and Imaging, Signal Processing, Image Security, Steganography, Image Processing.

\section{Keywords}

Watermarking, DWT, DRM, Copy Right Protection, RGB, YIQ.

\section{INTRODUCTION}

Due to rapid growth of multimedia applications over Internet, we have seen an explosion of data in the Internet and the extensive use of digital media. Consequently, digital data owners can transfer multimedia documents across the Internet easily. Therefore, there is an increase in the concern over copyright protection of digital content $[1,2]$. In the early days, encryption and control access techniques were employed to protect the ownership of media. However, to protect against unauthorized copying after the media have been successfully transmitted and decrypted, recently the watermarking techniques are utilized [3], because watermarking algorithms embed the watermark into digital data and using these watermark we can prevent the unauthorized copying.

A digital watermark is a pattern of bits inserted into an image, audio or video file. The name comes from the barely visible text or graphics imprinted on stationery that identifies the manufacturer of the stationery. There are several applications of watermarking such as, Broadcast monitoring, Owner identification, Proof of ownership, Transaction tracking, Content authentication, Copy control, and Device control [4, 5].

The watermarking techniques proposed in the literature fall in two categories: spatial-domain methods [4] and transformdomain methods [7, 8, 12, 14, 15 and 16]. Many techniques have been proposed in the spatial domain, such as the LSB (least significant bit) insertion method [9], the patchwork method [4] and the texture block coding method [4]. These techniques process the location and luminance of the image pixel directly. The LSB method has a major disadvantage that the least significant bits may be easily destroyed such as randomly flipping the lower bits or lossy compression.

A transform-domain method, such as the Fourier Transform [10], Discrete Cosine Transform, [11, 13], or Discrete Wavelet Transform $[12,14,15,16]$, are based on special transformations, and processes the coefficients in the frequency domain for hiding data. In these methods the watermark is hidden in the high frequency coefficients [12] or middle frequency coefficients [13] of the protected image. The low frequency coefficients are more likely to be suppressed by filtration as noise. Therefore, the high frequency coefficients of the protected image are used to embed the watermark [12]. How to select the best frequency portions of the image for hiding watermark is an important and difficult topic. The transform-domain method is more robust than the spatial-domain method against compression, cropping, and jittering [14]. The robustness is maintained at the price of imperceptibility in the transform domain.

In this paper, we propose a watermarking algorithm in which the color image is transformed into Red (R), Green (G) and Blue (B) channels. Further, these channels are transformed into Luminance (Y), Intensity (I) and Hue (Q) channels. The Discrete Wavelet Transformation (DWT) is applied on $\mathrm{Y}$ channel. The 
high and middle frequency components of this $\mathrm{Y}$ channel are modified to embed the watermark. The locations of modified frequency bands are stored into an array called key array. The inverse DWT is applied on this decomposed $\mathrm{Y}$ channel to get watermarked $\mathrm{Y}$ channel. The modified $\mathrm{Y}$, original I and Q are transformed into Color image. The high and middle frequency bands are used in embedding, because these bands contain the edge information of image, and human vision system is less sensitive to change in edge. In the extraction algorithm the watermarked image is transformed into RGB channels. The RGB channels are transformed into YIQ, and then the DWT is applied on $\mathrm{Y}$ channel. To extract the watermark the modified high and medium frequency components are selected using the key array. The correlation between extracted watermark and original watermark is calculated for objective judgment of the extraction fidelity. The use of key array in extraction algorithm helps to easily identify the frequency components used for embedding the watermark, thus increases the speed of extraction.

The paper is organized as follows: In Section 2, we describe the Discrete Wavelet Transformation. In Section 3, we describe the proposed watermark embedding extraction model. In Section 4, we present our results. Finally, in Section 5, we conclude our paper.

\section{DISCRETE WAVELET TRANSFORMATION}

The Discrete Wavelet Transform of an image divides the image into bands of approximately equal bandwidth on a logarithmic scale. This is similar to the retina of the human eye that splits the image into several components of each having a bandwidth of approximately equal to one octave. It is believed that the use of DWT for watermarking produces an imperceptible watermark [14].

The basic idea of the DWT for a two-dimensional image is described as follows. An image is first decomposed into four parts of high, middle, and low frequency subcomponents (i.e., $L L_{1}, H L_{1}, L H_{1}, H H_{1}$ ) by critically subsampling horizontal and vertical channels using subcomponent filters. The subcomponents labeled $H L_{1}, L H_{1}$, and $H H_{1}$ represent the finest scale wavelet coefficients. To obtain the next coarser scaled wavelet components, the subcomponent $L L_{1}$ is further decomposed and critically subsampled. This process is repeated several times, which is determined by the application at hand. An example of an image being decomposed into ten subcomponents for three levels is shown in Figure 1.

We used third level DWT decomposition which increases the Signal to Noise Ratio by reducing the effect of noise on cover image [16]. To embed the watermark high frequency components are considered, since high frequency components contains edge information and the human eye is less sensitive to changes in edges. In watermarking algorithms, the main concern besides invisibility of the watermark is how to choose the frequency components to embed the watermark such that it will survive the possible attacks that the transmitted image may undergo.

\section{PROPOSED MODEL}

The proposed DWT- scheme is a non-blind image watermarking scheme, where in it embeds the monochrome image (Logo) into a selected set of DWT coefficients. The embedding and extraction algorithms are shown in Figure $2 \mathrm{a}$ and Figure $2 \mathrm{~b}$, respectively.

\subsection{Watermark Embedding Algorithm}

As shown in Figure 2a the R, G, B channels are extracted from color image, and then these $\mathrm{R}, \mathrm{G}$, and $\mathrm{B}$ channels are transformed to Y, I, Q channels. To embed the watermark, only high frequency components of luminance channel of image are considered because high frequency luminance components are less sensitive to human eye.

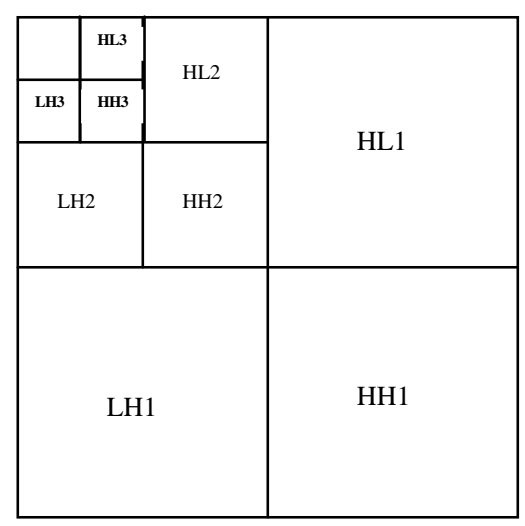

Figure 1: Third Level DWT Decomposition

The DWT is applied on Luminance channel of cover image, and watermark is embedded in high and middle frequency components. The location of the modified high frequency components are maintained in key array $K$. The inverse DWT is applied on watermarked Luminance channel, and then this channel is combined with Intensity and hue channels to get the watermarked color image. The watermark embedding algorithm can be summarized as follows:

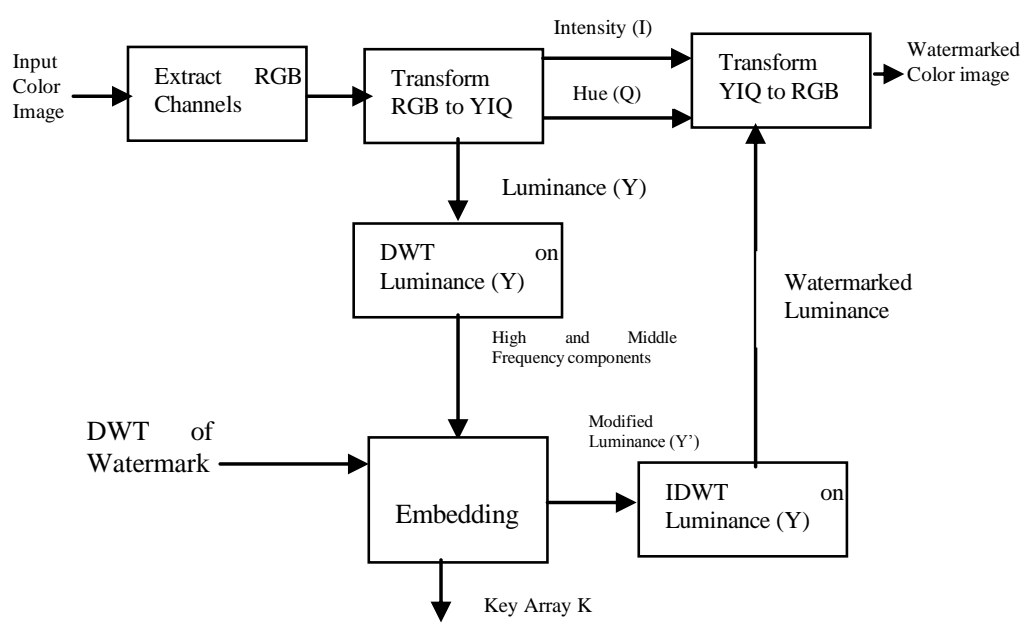

Figure 2a: Watermark Embedding Algorithm 


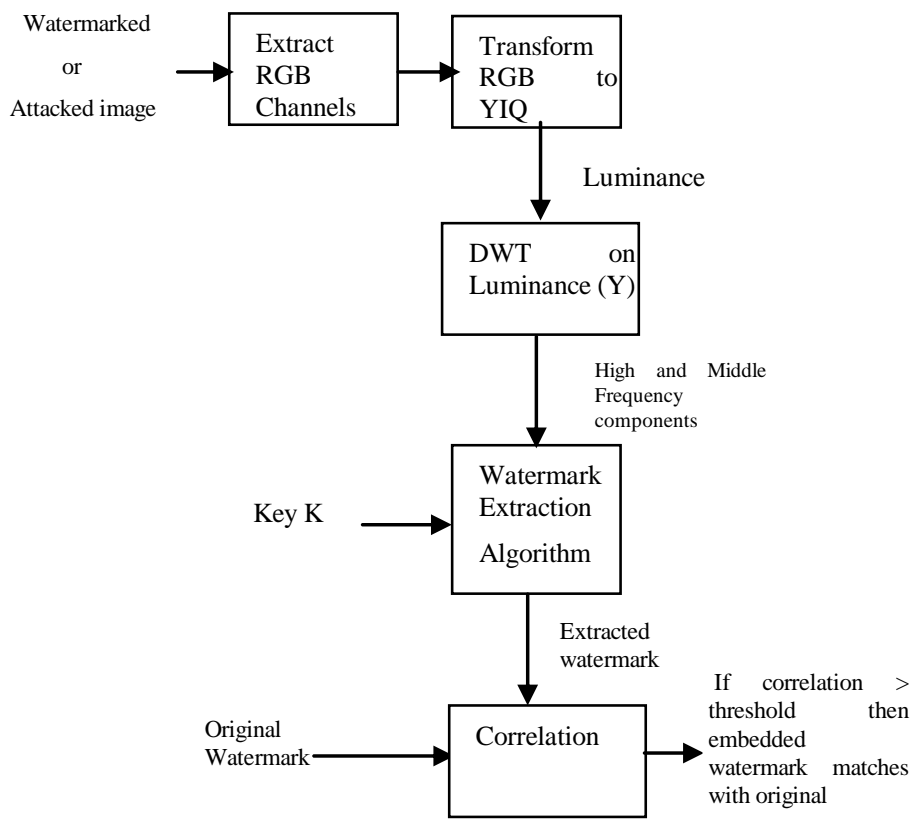

Figure 2b: Watermark Extraction Algorithm

Algorithm: Watermark Embedding

Input: cover image (color), watermark (monochrome) image.

Output: Watermarked color image.

1. Read color (cover) image $I$ of size $N \times N$.

2. Read the watermark (monochrome) image $X$ of size $M$ $\times M$ where $X(\mathrm{i}, \mathrm{j}) \in\{0,1\}, i=1,2, \ldots, M, j=1,2, \ldots$, $M$. Apply DWT on $X$ to get $D=\left\{d_{i j}\right\}$ of size $M \times M$.

3. Compute $R, G, B$ channels of size $N \times N$ from color image $I$.

4. Transform these $R, G, B$ channels into $Y, I, Q$ channels.

5. The frequency subcomponents $\left\{H H_{1}, H L_{1}, L H_{1},\left\{\left\{H H_{2}\right.\right.\right.$, $\left.\left.\left.H L_{2}, L H_{2},\left\{H H_{3}, H L_{3}, L H_{3}, L L_{3}\right\}\right\}\right\}\right\}$ are obtained by computing the third level DWT of the $Y$ channel

6. Embed the watermark components into the frequency subcomponents, starting from $H H_{1}$ for each row select the frequency coefficients in descending order with respect to their absolute values. Modify each frequency coefficient $f$ to $\quad f^{\prime}=f+\ll d_{i j}$,

Where $\propto$ is Watermark scaling factor, and $d_{i j}$ is watermark frequency coefficient. If the $H H_{1}$ subcomponent is not sufficient to embed the complete watermark, then go for next subcomponents in a sequence $\left\{L H_{1}, H L_{1},\left\{\left\{H H_{2}, H L_{2}, L H_{2},\left\{H L_{3}, L H_{3}\right.\right.\right.\right.$, $\left.\left.\left.\mathrm{HH}_{3}\right\}\right\}\right\}$.

7. Save the location of the modified frequency components into a key array $K$ of size $N \times N$. The key array $K$ has value one if the coefficient is modified and zero if not.

8. Replace $\{f\}$ by $\left\{f^{\prime}\right\}$ in decomposed $Y$ channel.

9. Compute Inverse DWT of modified $Y$ channel.

10. Combine modified $Y$ channel with $\mathrm{I}$ and $\mathrm{Q}$ to get watermarked color image

\section{Input:}

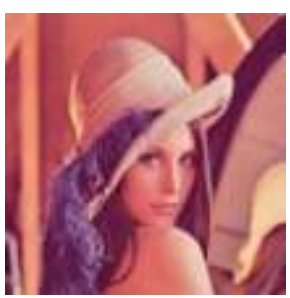

Figure 3a: Original Image

\section{NITW}

Figure 3b: Watermark image

\section{Output:}
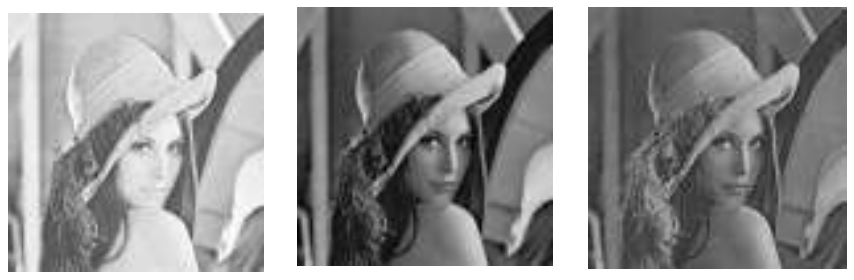

Figure 3c: Red, Green and Blue Components of color image
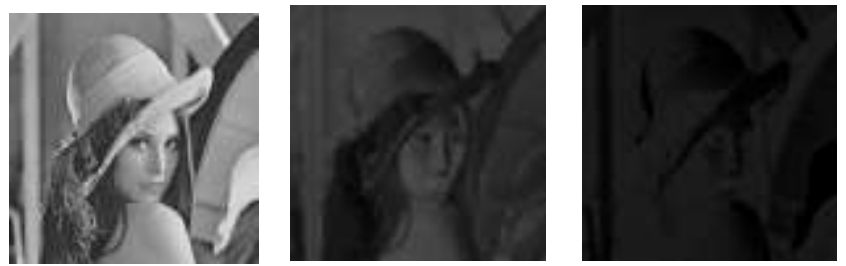

3d: Luminance, Intensity and Hue Components of color Image

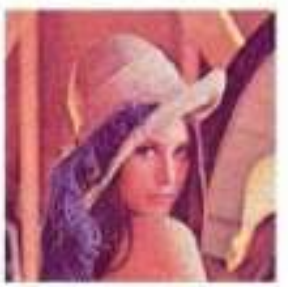

Figure 3e: Watermarked Color image

\subsection{Watermark Extraction Algorithm}

As shown in Figure $2 b$ the R, G, B channels are extracted from the watermarked color image. These channels are transformed into Y, I, Q channels. To extract the watermark, only luminance channel of image is considered. The DWT is applied on Luminance channel of image, and from this, only the modified high and middle frequency components are retrieved using the key array $K$. These frequency components are used to extract the watermark. The correlation between extracted watermark and available watermark is calculated. If the extracted watermark and 
available watermark are tightly correlated then it indicates the embedded watermark matches with original watermark.

The watermark extraction algorithm can be summarized as follows:

\author{
Algorithm: Watermark Extraction \\ Input: Watermarked image, cover (color) image \\ Output: Watermark
}

1. Read the Watermarked image $I^{\prime}$ of size $N \times N$.

2. Compute $R^{\prime}, G^{\prime}, B^{\prime}$ channels of size $N \times N$ from watermarked color image $I^{\prime}$.

3. Transform these $R^{\prime}, G^{\prime}, B^{\prime}$ channels into $Y^{\prime}, I^{\prime}, Q^{\prime}$ channels.

4. The frequency subcomponents $\left\{H H_{1}, H L_{1}, L H_{1},\left\{\left\{H H_{2}\right.\right.\right.$, $\left.\left.\left.H L_{2}, L H_{2}, \quad\left\{H H_{3}, H L_{3}, L H_{3}, L L_{3}\right\}\right\}\right\}\right\}$ are obtained by computing the third level DWT of the $Y^{\prime}$ channel

5. The frequency subcomponents $\left\{H H_{1}, H L_{1}, L H_{1},\left\{\left\{H H_{2}\right.\right.\right.$, $\left.\left.\left.H L_{2}, L H_{2},\left\{H H_{3}, H L_{3}, L H_{3}, L L_{3}\right\}\right\}\right\}\right\}$ are obtained by computing the third level DWT of the $Y$ channel of the unwatermarked color image.

6. Extract the watermark bits from the frequency subcomponents, starting from $H H_{1}$ and using key array $K$ as $X_{\mathrm{ij}}{ }^{\prime}=\left(f^{\mathrm{c}}-f\right) \propto$.

If $X_{\mathrm{ij}}{ }^{\prime}>T$, then $X_{\mathrm{ij}}{ }^{\prime}=1$ else $X_{\mathrm{ij}}{ }^{\prime}=0$, Where $i=1,2, \ldots$, $M$, and $j=1,2, \ldots, M, f=$ frequency coefficient of $Y$ at the corresponding level and subcomponent, $f^{\prime}=$ frequency coefficient of $Y^{\prime}$ at the corresponding level and subcomponent, $T$ is between 0 and 1 and $\alpha$ is scaling factor.

Input:

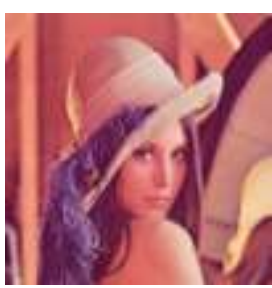

Figure 4a Original Image

Output:
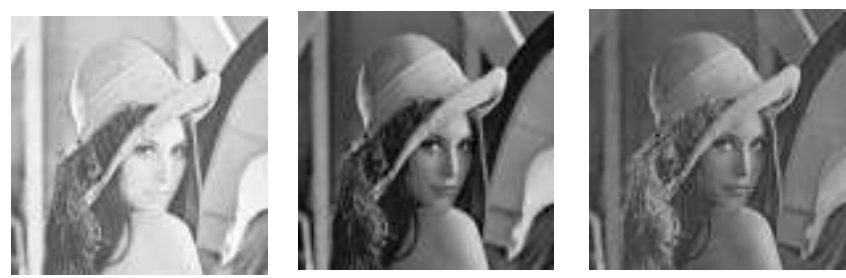

Figure 4c: Red, Green and Blue Components of Watermarked image Image
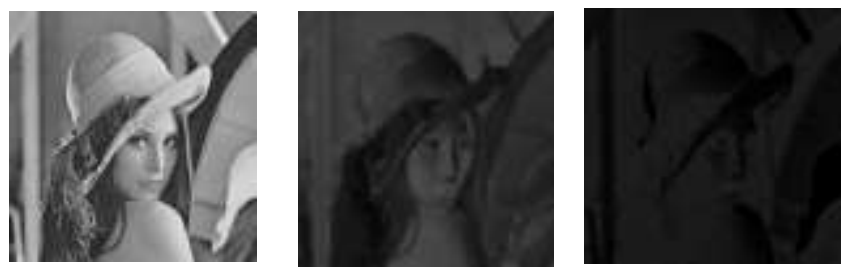

Figure 4d: Luminance, Intensity and Hue Components of watermarked image

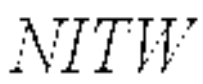

Figure 4e: Extracted Watermark

\section{RESULTS AND DISCUSSIONS}

To test the algorithm, the MATLAB modules are defined separately for embedding and extraction of watermark. A 256 256 sized color image of Lena is taken as a cover image and the monochrome logo pattern of size $60 \times 70$, as a watermark. Various experiments are conducted on watermarked image to test the robustness of algorithm. Also, image processing operations like cropping, filtering, compression and geometric transformations are applied on watermarked image. Then the quality of extracted watermark is compared with original watermark using Normalized Correlation. The performance of embedding algorithm is measured using Signal to Noise Ratio metric.

\subsection{Robustness against Image Cropping}

Image cropping is applied on watermarked image by cropping $20 \%$ of image. This is shown in Figure 5a.Then the extraction algorithm is applied to this cropped image. The extracted watermark is shown in the Figure $5 \mathrm{~b}$. The robustness of the algorithm is tested by applying different percentage of cropping. It is found that the quality of extracted watermark decays as the percentage of cropping increases. We get good quality of extracted watermark if the cropping is less than $40 \%$ as shown in

Figure 4b : Watermarked

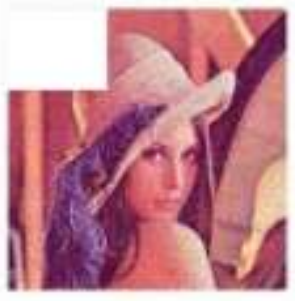
Figure 5c.

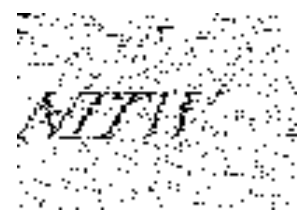

Figure 5a: Cropped image

Figure 5b: Extracted

Watermark after cropping 


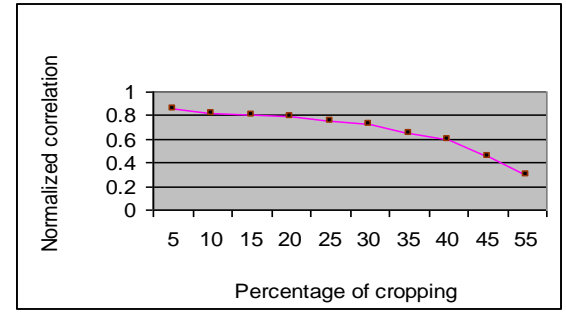

Figure 5c: Effect of cropping

\subsection{Robustness against Compression}

We use JPEG compression to check the robustness of algorithm against lossy compression. As shown in Figure 6, the quality of compressed image purely depends on the value of compression ratio. If the value of compression ratio is less than 6 , then the normalized correlation between extracted and original watermark is nearly 0.8 , thus image quality is also good. As the compression ratio increases the quality of the extracted watermark decays. The correlation coefficient is closer to 1 .

\subsection{Robustness against Filtering}

Image filtering is one of image processing method that is applied to remove the noise. Gaussian filters are important in many signal processing, image processing, and communication applications. These filters are characterized by narrow bandwidths, sharp cutoffs, and low overshoots. A key feature of Gaussian filters is that it has the same response shape in both time and frequency domains. The Gaussian filter is applied on the watermarked image. The filtered image is as shown in Figure 7a. The extracted watermark is extracted from this filtered image is shown in Figure 7b. The Normalized correlation between extracted watermark and original watermark is 0.878 .This represent the algorithm is robust against filtering

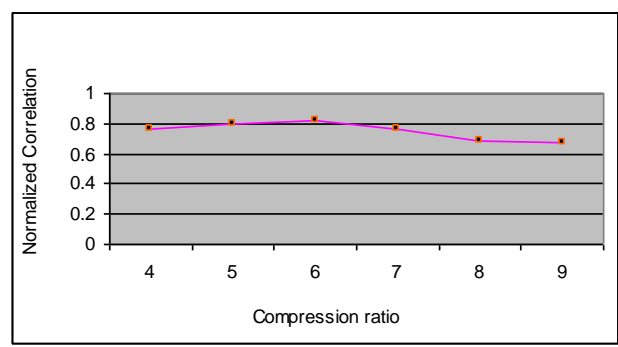

Figure 6: Effect of Compression
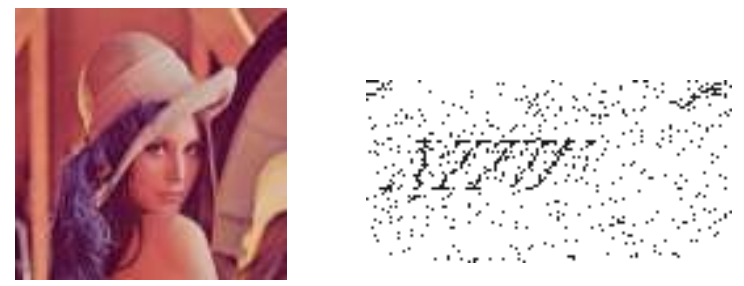

Figure 7a: Low-Pass filtered

Figure 7b: Extracted

Watermarked image Watermark

\subsection{Robustness against Geometrical Transformation}

The geometric transformations are important manipulations of image. The transformations that modify the size of image is known as scaling, and the transformations that modify the angle of orientation is known as rotation. Geometrical transformation affects the quality of extracted watermark. The effect of different transformation on the watermarked image by measuring the Normalized correlation between the original watermark and extracted watermark is shown in Figure 8 to Figure 10. For the effect of scaling, we found the correlation is consistent for the scale factor of range 0.10 to 0.30 , for the effect of rotation, we found the correlation is good for the rotation angle must in the range 1 to 14 degrees .

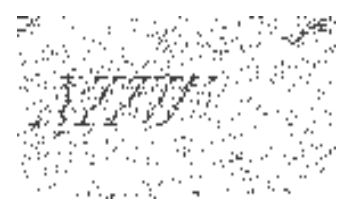

Figure 8: Extracted watermark from 0,2 scaled watermarked image and correlation is nearly 0.7705

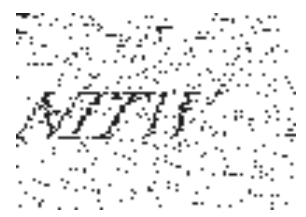

Figure 9: Extracted watermark from 6 degree Rotation of Watermarked image and correlation is nearly 0.7854

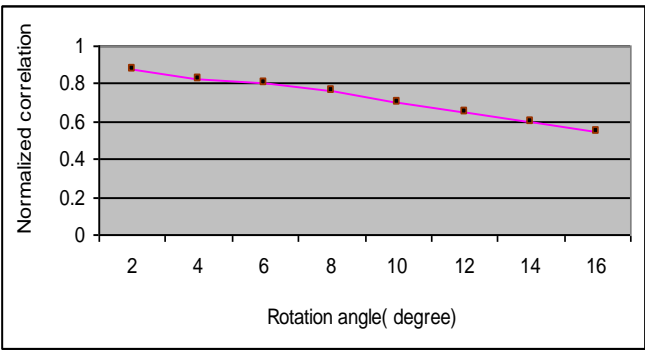

Figure 10: Effect of Rotation

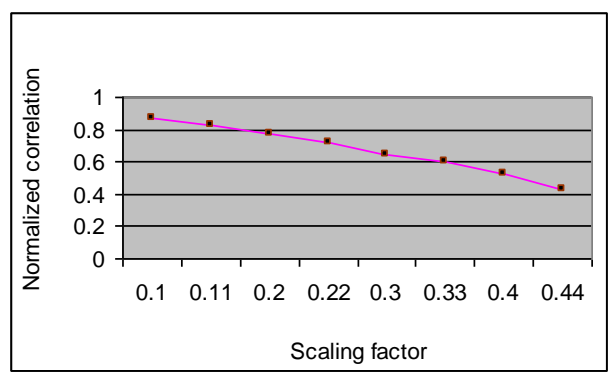

Figure 11: Effect of Scaling 


\subsection{Peak Signal to Noise Ratio}

In experiments, we obtained the third level DWT decomposition using the Haar filter with the value of $\alpha=0.6$. We calculated the amount of noise added into the color (cover) image using Peak Signal to Noise (PSNR) metric.

$$
P S N R=10 \log _{10} \frac{R \times R}{M S E}
$$

Where $R=$ maximum fluctuation in the input image $=255$,

$M S E=\sum_{i=1}^{m} \sum_{j=1}^{n} \sum_{k=1}^{3} \frac{\left(W(i, j)_{k}-W^{\prime}(i, j)_{k}\right)^{2}}{3 m n}$

$m=$ height of image, $n=$ width of image, $W(i, j)_{k}$ and $W^{\prime}(i, j)_{k}$ represent $\mathrm{R}, \mathrm{G}, \mathrm{B}$ channels of unwatermarked color image and Watermarked color image respectively. We found that the value of Peak Signal to Noise ratio is $48.52 \mathrm{~dB}$. After extracting the watermark, it is compared with the original watermark by calculating Normalized correlation that is defined below:

$$
\text { Normalized Correlation }=\frac{\sum_{i} \sum_{j} W(i, j) W^{\prime}(i, j)}{\sum \sum_{W(i, j)^{2}}}
$$

Where $W(i, j)=$ original watermark and $W^{\prime}(i, j)=$ Extracted watermark.

\subsection{Efficiency of Algorithm}

The determination of increase in the detection speed and efficiency of the algorithm, the location of modified high and middle frequency components are stored into key array. Use of this key array improves the speed of the extraction algorithm. Table 1 shows the time taken by algorithm to extract the watermark with and without key array. Thus by using the key array the speed of extraction is improved.

Table 1: Performance of extraction algorithm with and without key array

\begin{tabular}{|c|c|}
\hline \multicolumn{2}{|c|}{ Time taken by extraction algorithm (in seconds). } \\
\hline Without Key Array & With Key Array \\
\hline 29.12 & 19.76 \\
\hline
\end{tabular}

\subsection{Comparison}

We compare the performance of our algorithm with the other watermarking algorithms based on DWT [12, 15]. The comparison is decided in Table 2.

Table 2: Comparison of proposed algorithm with other DWT

\begin{tabular}{|c|c|c|c|}
\hline Properties & $\begin{array}{c}\text { Ersin } \\
\text { Elbasi[12] }\end{array}$ & $\begin{array}{c}\text { Ersin Elbasi } \\
{[15]}\end{array}$ & Proposed Algorithm \\
\hline $\begin{array}{l}\text { Cover } \\
\text { Data }\end{array}$ & Gray Scale & $\begin{array}{l}\text { Luminance(y) } \\
\text { channel } \\
\text { of video frame }\end{array}$ & $\begin{array}{l}\text { Luminance }(y) \\
\text { channel } \\
\text { of color image }\end{array}$ \\
\hline $\begin{array}{l}\text { Domain of } \\
\text { embeddin } \\
\mathrm{g}\end{array}$ & $\begin{array}{l}\text { Frequency } \\
\text { domain } \\
\text { DWT }\end{array}$ & $\begin{array}{c}\text { Frequency } \\
\text { domain DWT }\end{array}$ & $\begin{array}{c}\text { Frequency domain } \\
\text { DWT }\end{array}$ \\
\hline $\begin{array}{l}\text { Frequency } \\
\text { bands }\end{array}$ & $\begin{array}{c}2 \text { bands } \\
\{L L, H H\}\end{array}$ & $\begin{array}{c}\text { All } 4 \\
\text { frequency } \\
\text { components } \\
\{\text { LL,LH,HL,H } \\
\text { H }\}\end{array}$ & $\begin{array}{c}\text { High frequency and } \\
\text { middle components } \\
\{\text { HL, } \mathrm{LH}, \mathrm{HH}\}\end{array}$ \\
\hline $\begin{array}{l}\text { Watermar } \\
\quad \mathbf{k}\end{array}$ & $\begin{array}{l}\text { Pseudo } \\
\text { random } \\
\text { Number } \\
(\text { PRN) }\end{array}$ & Binary image & $\begin{array}{c}\text { Monochrome image } \\
(\operatorname{logo})\end{array}$ \\
\hline $\begin{array}{l}\text { PSNR Of } \\
\text { watermar } \\
\text { ked image }\end{array}$ & $40.86 \mathrm{~dB}$ & $42.43 \mathrm{~dB}$ & $48.32 \mathrm{~dB}$ \\
\hline$\alpha$ & $\begin{array}{l}0.1 \text { for } L L \\
\text { and .0.4 for } \\
\text { HH }\end{array}$ & ---------- & 0.6 \\
\hline $\begin{array}{l}\text { Input to } \\
\text { extraction } \\
\text { algorithm }\end{array}$ & $\begin{array}{c}T 1=100, \\
T 2=110 \\
\text { for } L L \text { and } \\
T 1=30, \\
T 2=40 \text { for } \\
\text { HH }\end{array}$ & $\begin{array}{l}\text { Cover video } \\
\text { frame }\end{array}$ & $\begin{array}{c}\text { Key array } K \\
\text { Color (Cover) image }\end{array}$ \\
\hline
\end{tabular}
algorithm

As shown in the Table 2, the proposed algorithm has PSNR of $48.32 \mathrm{~dB}$, which is higher than the algorithms used in $[12,15]$. If the watermark is embedded into low frequency components, the low frequency components are more likely to be suppressed by filtration as noise. Hence our algorithm embeds the watermark into high and middle frequency components, which improved the robustness of algorithm against attacks and the visual quality of watermarked color images is same as original image. The key array used in extraction algorithm helps to easily identify the modified frequency components and thus improves the speed of extraction. 


\section{CONCLUSION}

In this paper we presented a new method of embedding watermark into color image. The luminance component of image is considered for embedding watermark. On this luminance component of image DWT is applied to decompose this image into high and middle frequency components. Combinations of high and middle frequency components are considered for watermarking, because the high frequency contains edge information. Further, human eyes are less sensitive to change in the edge. The strength of algorithm is tested using different types of image processing attacks, like image cropping, image filtering, image compression etc. algorithm is found rigid to different types of attack.

\section{REFERENCES}

[1] A. Piva, F. Bartolini, and M. Barni, "Managing copyright in open networks". IEEE Internet Computing, Vol. 6, Issue 3, pp: 18 -26, May-June 2002.

[2] Chun-Shien Lu, Hong-Yuan, and Mark Liao, "Multipurpose Watermarking for Image Authentication and Protection". IEEE Transactions on Image Processing, Vol. 10, Issue 10, pp: 1579 -1592, Oct 2001.

[3] Joo Lee and Sung-Hwan Jung, "A survey of watermarking techniques applied to multimedia". Proceedings 2001, IEEE International Symposium on Industrial Electronics (ISIE2001), Vol. 1, pp. 272 -277, 2001.

[4] I. J. Cox, M. L. Miller, and J. A. Bloom, "Digital Watermarking", Morgan Kaufmann Publishers, 2002.

[5] R. G. Schyndel, A. Tirkel, and C. Osborne, "A Digital Watermark," Proceedings of 1994 International Conference on Image Processing (ICIP 1994), Austin, Texas, November 13-16, pp. 86-90, 1994.

[6] C.T. Hsu. and J:L. Wu, "Hidden digital watermarking in images." IEEE Transaction on Image Processing, Vol. 8, No. 1, pp. 58-68, Jan. 1999.
[7] M. Barni, E Bartolini, V. Cappellini, and A. Piva, "A DCT- domain system for robust image watermarking," Signal Processing Vol. 66, No. 3, pp. 357-372, May 1998.

[8] C. T. Hsu. and 1. L.Wu, "DCT-based watermarking for video," IEEE Transactions on consumers electronics, Vol. 44, No.1 , pp. 206-216, Feb. 1998.

[9] S. Saha, and R. Vcmuri, "How do image statistics impact lossy coding Performance?" Information Technology, pp. 42-47, 2000.

[10] P,-L. Lin," Robust transparent image watermarking system with spatial mechanisms", The Journal of Systems and Software, pp.107-116. 2000.

[11] J. J. K. O'Ruanaidh, W. J. Dowling, and E M. Boland, "Phase watermarking of digital images," Proceedings of IEEE International conference on Image Processing, Vol. 3, pp. 239-242, Sep. 1996.

[12] Ersin Elbasi and Ahmet M. Eskicioglu, “ A DWT-Based Robust Semi-Blind image Watermarking Algorithm Using Two bands" Proc. SPIE, Vol. 6072, 2006.

[13] Chiou-Ting Hsu and Ja-Ling Wu, "Hidden Digital watermarks in Images" IEEE Transactions on Image Processing, Vol. 8, no. 1, January 1999.

[14] Peter Meerwald and Andreas Uhl, "A Survey of Waveletdomain Watermarking Algorithms", Security and Watermarking of Multimedia Contents III, Proceedings SPIE Vol. 4314, pp. 505-516. January 20 - 26, 2001

[15] Ersin Elbasi and Ahmet M. Eskicioglu ,"Robust DWT Based MPEG-1 Watermarking in Four Bands" The Second Secure Knowledge Management Workshop (SKM), New York City, NY, September 28-29, 2006.

[16] Suhad Hajjara, Moussa Abdallah, Amjad Hudaib, "Digital Image Watermarking Using Localized Biorthogonal Wavelets" European Journal of Scientific Research, ISSN 1450-216X Vol.26 No.4, pp.594-608, 2009. 